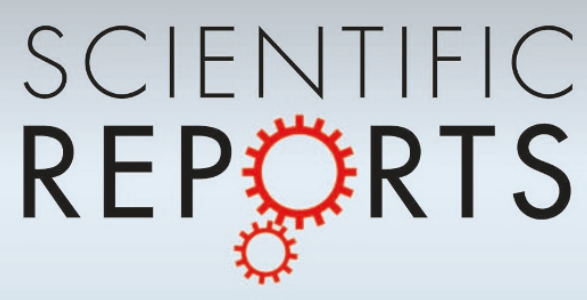

OPEN

SUBJECT AREAS:

ELECTRONIC DEVICES

MECHANICAL AND STRUCTURAL PROPERTIES AND DEVICES

Received

7 November 2013

Accepted

13 February 2014

Published

28 February 2014

Correspondence and requests for materials should be addressed to

H.Z.W. (wanghz@ dhu.edu.cn) or Y.G.L. lyaogang_li@dhu.edu.

\section{Highly Strong and Elastic Graphene Fibres Prepared from Universal Graphene Oxide Precursors}

\author{
Guoji Huang' ', Chengyi Hou', Yuanlong Shao', Hongzhi Wang', Qinghong Zhang', Yaogang Li \\ \& Meifang Zhu'
} 'State Key Laboratory for Modification of Chemical Fibers and Polymer Materials, College of Materials Science and Engineering,
Donghua University, 201620 (People's Republic of China), '2Engineering Research Center of Advanced Glasses Manufacturing
Technology, College of Materials Science and Engineering, Donghua University, 201620 (People's Republic of China).

Graphene fibres are continuously prepared from universal graphene oxide precursors by a novel hydrogel-assisted spinning method. With assistance of a rolling process, meters of ribbon-like GFs, or GRs with improved conductivity, tensile strength, and a long-range ordered compact layer structure are successfully obtained. Furthermore, we refined our spinning process to obtained elastic GRs with a mixing microstructure and exceptional elasticity, which may provide a platform for electronic skins and wearable electronics, sensors, and energy devices.

raphene is a single-atom-thick two-dimensional (2D) material, comprised of a monolayer of hexagonally arranged $\mathrm{sp}^{2}$-hybridized $\mathrm{C}$ atoms. It has received much interest, because of its high electron mobility, thermal conductivity, elasticity, and stiffness ${ }^{1-5}$. These properties make graphene attractive for application in nanoelectronic devices, sensors, functional composites, and energy storage. To maximize the practical application of graphene, it is important to integrate individual graphene nanosheets into ordered macroscopic materials. Nowadays, graphene sheets can be easily assembled into macroscopic papers and films $s^{6-8}$. The poor dispersibility of pristine graphene in common solvents and the variable layer-by-layer stacking of graphene sheets have also been overcome to prepare ordered graphene fibres $(\mathrm{GFs})^{9-11}$. Several methods have been reported to prepare ordered GFs, such as hydrothermal strategies and wet spinning of a concentrated graphene oxide (GO) liquid crystal solution. However, the electrical conductivity and mechanical strength of the as-obtained GFs are unsatisfactory for practical applications in lightweight chemical supercapacitors, sensors, stretchable electronics and other energy devices ${ }^{12-16}$. Size selection of GO sheets, Ag doping, and thermal treatment of GFs have been probed to prepare highly ordered and reduced GFs, in an attempt to improve GFs' electrical and mechanical properties ${ }^{17-20}$. However, these methods are complex and costly. Additionally, the stretching properties of the asobtained GFs remain inferior to those of conventional organic fibers ${ }^{21-23}$. Therefore, developing a straightforward and effective method for improving GFs' performance is still desirable.

In this study, we controlled the GFs microstructure without enhancers or selection of large graphene sheet sizes, and greatly improved the GFs properties. A self-assembly method was used to prepare the GFs. With assistance of a rolling machine, we further fabricated ribbon-like GFs, or graphene ribbons (GRs), with a longrange ordered compact layer structure. The electrical conductivity and tensile strength of GRs were both enhanced compared to those of GFs. Elastic GRs (EGRs) with high ultimate failure strain can also be obtained. Our processes provide a new approach to prepare pure graphene-based fibres with enhanced performance, and new opportunities for their applications in flexible and stretchable nanoelectronics, such as wearable electronics and energy devices.

To prepare GFs, GO fibres (GOFs) were firstly prepared by spinning a GO hydrogel, as shown schematically in Fig. 1a. GO suspensions (Supplementary Fig. S1a online) were mixed with sodium deoxycholate (NaDC). The obtained NaDC-functionalized GO sheets dispersed well in a NaDC-hydrated matrix and formed a spinnable supramolecular hydrogel (Supplementary Fig. S1b online). When the spun GO hydrogel was immersed in ethanol, $\mathrm{NaDC}$ dissolved and the GO sheets assembled to form a GOF due to van der Waals interactions. Spinning a $30 \mathrm{mg} / \mathrm{mL}$ aqueous $\mathrm{GO}$ suspension containing a desired ratio of $\mathrm{NaDC}$ (5-9\%, weight- ${ }_{\mathrm{NaDC}} /$ weight- ${ }_{\text {water }}$ ) yielded continuous fibers. This spinning process is shown in Supplementary Movie S1 online. Meter lengths of 


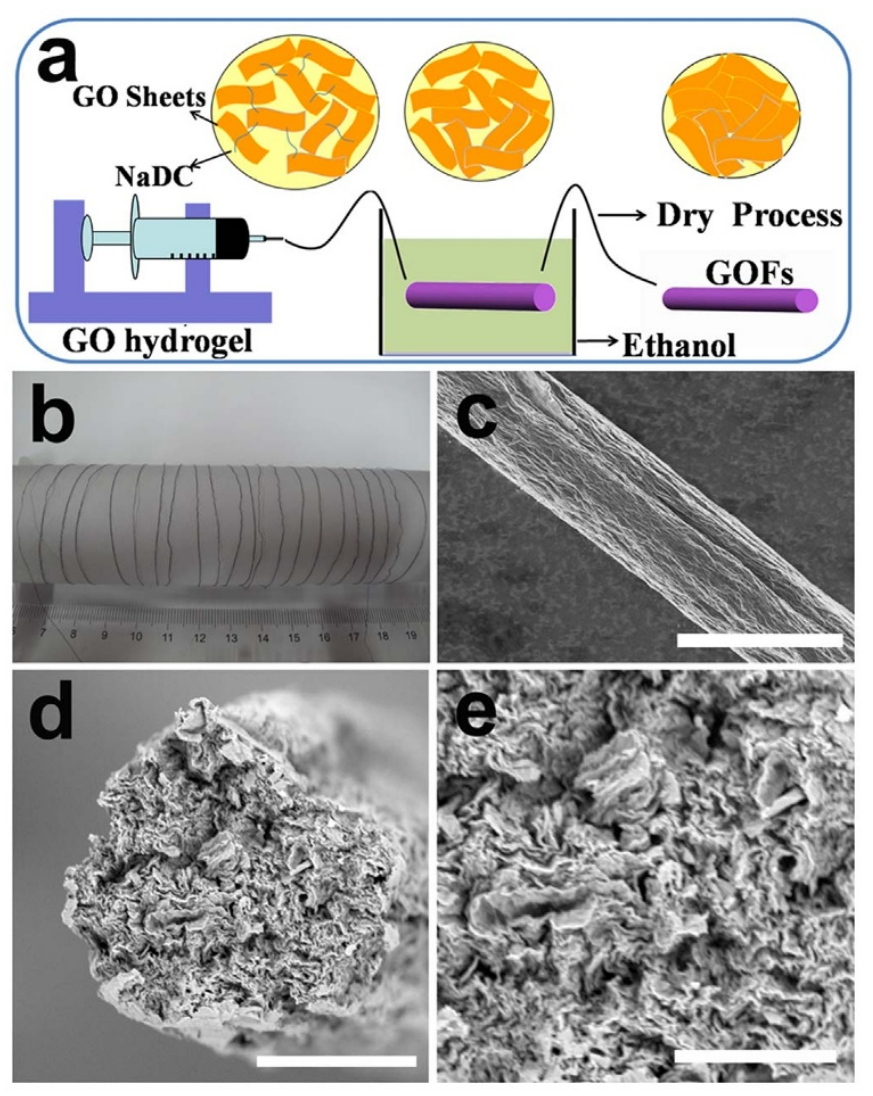

Figure $1 \mid$ (a) Schematic diagram of GOF fabrication. (b) Photograph of 3$\mathrm{m}$-long GFs wound on a drum (diameter, $4 \mathrm{~cm}$ ). (c) and (d) FE-SEM images of top and cross-section view of a GF, respectively. (e) High magnification FE-SEM image of cross-section view of a GF. Scale bars: $50 \mu \mathrm{m}, 20 \mu \mathrm{m}$ and $10 \mu \mathrm{m}$, respectively. Figures were drawn by Guoji Huang.

GOFs with tunable diameters $(10-60 \mu \mathrm{m})$ were prepared using polytetrafluoroethylene (PTFE) pipelines with different diameters. Finally, GOFs were reduced to GFs after being treated with HI. Meters of GFs can be prepared through above processes (Fig. 1b). The Fourier transform infrared spectra, Raman spectra, and X-ray diffraction (XRD) patterns of NaDC, GO hydrogel, GOFs, and GFs are shown in Supplementary Fig. S2 online. Both dissolution of $\mathrm{NaDC}$ and reduction of GO could be confirmed by the spectroscopic results $^{24-27}$.

\section{Results}

The morphology and structure of GFs were analyzed by field emission-scanning electron microscopy (FE-SEM). In GFs, graphene sheets were densely stacked and largely aligned to the fiber axis, which is similar to previously reported graphene fibres ${ }^{9,28}$. This is attributed to the flowing GO hydrogel and the effect of the PTFE pipeline or capillary-induced shear force induced in wet spinning. GFs did not break during their knotting into a loop (Supplementary Fig. S3 online), demonstrating an excellent flexibility compared to CNT fibers ${ }^{29-31}$. The high-resolution cross-sectional FE-SEM image of GFs clearly shows that reduced GO sheets assembled randomly in GFs (Fig. 1c-e). The mean measured electrical conductivity of GFs reaches $3.08 \times 10^{4} \mathrm{~S} / \mathrm{m}$. A typical stress strain curve is shown in Fig. $2 b$. The mean experimentally determined tensile strength and elongation to break of the GF sample with best electrical properties are $238 \mathrm{MPa}$ and $2 \%$, respectively. The high tensile strength could be attributed to the directionally aligned structure and strong interactions between stacked reduced GO sheets. The tensile strength is higher than that of neat wet-spun single-walled CNT fibers (50$150 \mathrm{MPa}$ ) and GFs fabricated by other methods ${ }^{9-11,32,33}$. The average density of GFs was measured as $1.7960 \mathrm{~g} / \mathrm{cm}^{3}$, as a consequence, the specific strength and the average specific conductivity of the GFs is up to $132 \mathrm{kN} \cdot \mathrm{m} / \mathrm{kg}$ and $16 \mathrm{~S} \cdot \mathrm{m}^{2} / \mathrm{kg}$, respectively.

Based on the supramolecular hydrogel-assisted spinning method, we next fabricated GRs, or ribbon-like GFs, with assistance of a double roller crusher. During this process, a geometric confinement was induced by rolling, which leaded to long-range ordered structures of GRs. A schematic of the fabrication process is shown in Fig. 2a.

The length of PTFE pipeline (internal/external diameter of 300/ $700 \mu \mathrm{m})$ containing GO hydrogel was pressed in the double-roller crusher. GO hydrogel was subsequently released into a $95 \mathrm{wt} \%$ ethanol solution to dissolve the NaDC and yield a GOF with layered structure. After drying and reduction, $1.5 \mathrm{~m}$-long GRs with a uniform spiral structure were obtained (Fig. 2c). The thickness of GRs could be turned $(0.5-2.5 \mu \mathrm{m})$ through adjusting the distance between the two rollers $(300-400 \mu \mathrm{m})$. Raman spectra and XRD patterns of the resulting GRs are shown in Supplementary Fig. S2 online.

FE-SEM was used to characterize fibre samples. Fig. 2d demonstrates good flexibility of GRs, which is comparable to that of GFs. Surface of GRs is much more smooth than that of GFs (Fig. 2e), which could be attributed to the ordered reduced GO layers in GRs. This structure is confirmed by cross-sectional images shown in Fig. $2 \mathrm{f}-\mathrm{h}$. These compact layers are mainly attributed to the shear flow of the GO hydrogel, which arose from the double-roller crusher. The mechanism can be explained as follows: The GO hydrogel was injected into the PTFE pipeline at a certain rate, and GO sheets with a lamellar structure were interconnected to form a partially ordered structure along the axial direction ${ }^{9,17,34-36}$; subsequently, the PTFE pipeline containing GO hydrogel was pressed by the double-roller crusher, GO sheets were further rearranged as a result of the continuous compression force. Rounded fibers with a partially ordered structure (Fig. 1d) were then pressed into ribbon-like ones with a long-range ordered compact layer structure (Fig. 2f).

The GRs have a mean experimentally determined electronic conductivity of $5.7 \times 10^{4} \mathrm{~S} / \mathrm{m}$ and a tensile strength of $404 \mathrm{MPa}$ (Fig. 2b), which are about two times larger than those of our GFs, and are comparable to those of previously reported GFs (see Supplementary Table S1 online). In addition, the average density $\left(1.8355 \mathrm{~g} / \mathrm{cm}^{3}\right)$, specific strength $(220 \mathrm{kN} \cdot \mathrm{m} / \mathrm{kg})$ and specific conductivity $\left(32 \mathrm{~S} \cdot \mathrm{m}^{2} / \mathrm{kg}\right)$ of GRs are also higher than those of GFs. The above results indicate that ordered structure could remarkably improve the electrical and mechanical properties of GFs. However, their ultimate strain (2.25\%) maintains at the same level as previous GFs, which is still the weakness of nowadays GF products.

Next, we refined the GR manufacturing process to obtain elastic GRs. During the synthesis of GRs, after rolling, we reduced the ribbon-like GOFs before drying. Water molecules in wet GOFs gave space for movement of GO sheets to unregularly restack in the reduction reaction. Partially disordered structure was thus randomly formed. Fig. 3a shows the deformation of EGRs with an original length of about $10 \mathrm{~cm}$. The elastic fibre could be stretched to about $18 \mathrm{~cm}$ without breakage. After relaxation, it retracted immediately. Supplementary Movie S2 online shows this process. In the video, it is clearly seen that the EGRs behaved like a spring. We believe that the key factor which makes GRs elastic is the partially disordered "defects" induced in their ordered layer structure. They could lead to non-uniform shrink in the thickness direction of the fibre during drying, which twisted the GRs (see Fig. 3b and 3c). With mixing microstructures of GFs and GRs (Fig. 3d and 3e), EGRs had similar spectroscopic features to them (Supplementary Fig. S2 online). The mean measured tensile strength and conductivity of EGRs reaches $102 \mathrm{MPa}$ (Fig. 4a) and $3.55 \times 10^{4} \mathrm{~S} / \mathrm{m}$ (Fig. $4 \mathrm{~b}$ inset, black line), 


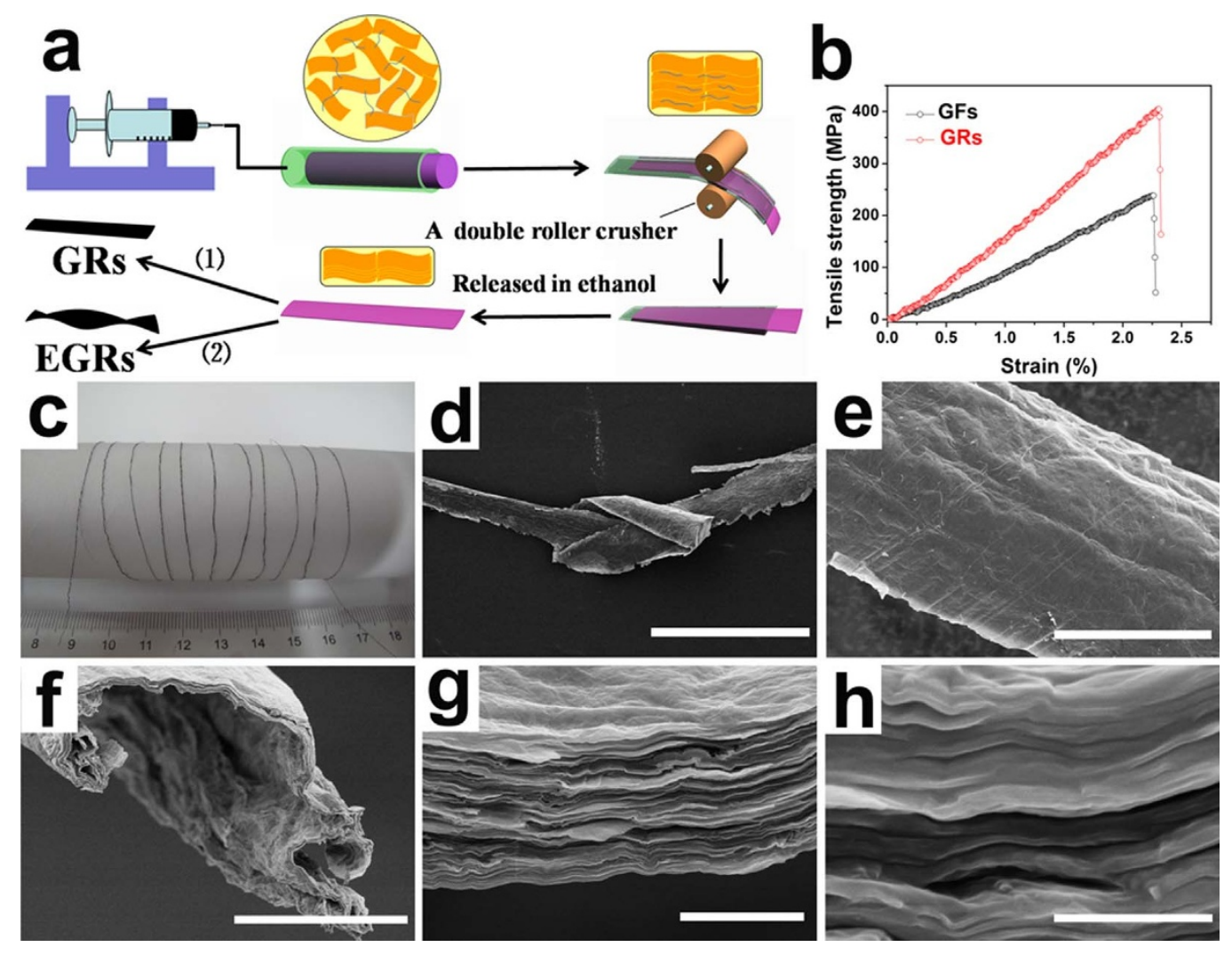

Figure $2 \mid$ (a) Schematic showing the fabrication of GRs and EGRs. Process (1) involves drying followed by reduction of the layered GOF structure to GRs, and (2) involves the reduction of the layered GOF structure, and then drying to yield EGRs. (b) Stress-strain curves of the GFs (black) and GRs (red). (c) Photograph of 1.5-m-long GRs wound on a drum (diameter, $4 \mathrm{~cm}$ ). FE-SEM images of (d) a tightened knot, (e) surface morphology, and (f) cross-sectional morphology of GRs. (g) and (h) are high-magnification images of (c). Scare bars: $1 \mathrm{~mm}, 50 \mu \mathrm{m}, 50 \mu \mathrm{m}, 2 \mu \mathrm{m}$ and $500 \mathrm{~nm}$, respectively. Figures were drawn by Guoji Huang.

respectively. The average density of EGRs is $1.5420 \mathrm{~g} / \mathrm{cm}^{3}$. The low density could be attributed to the partially disordered "defects" structure, which leads to a relatively lower specific strength $(66 \mathrm{kN} \cdot \mathrm{m} / \mathrm{kg})$ and specific conductivity $\left(22 \mathrm{~S} \cdot \mathrm{m}^{2} / \mathrm{kg}\right)$ compared to those of GRs. We further investigated the electrical resistance (R) during stretching. As shown in Fig. 4b, the electrical resistance of our EGR remained stable during the stretching and recovery process. Typical I-V curves of the EGR before (black) and after (red) stretching were overlapped (Fig. $4 \mathrm{~b}$ inset). This differs from traditional materials whose resistances are greatly influenced by their shape.
Furthermore, it is worth noting that the ultimate strain of EGRs is as high as $85 \%$, more than 6 times larger than the best result ever reported ${ }^{37}$. Fig. $4 \mathrm{c}$ presents cyclic stress-strain curves for the overall elastic property of the EGR. The fibre was stretched by the two ends with different predefined tensile strain from $10 \%$ to $70 \%$ for this measurement. The EGR shows a typical elastomeric behavior at $\varepsilon$ $\leq 50 \%$. Residual strain appears at higher strains $(\varepsilon \geq 60 \%)$. These results are similar to those of the elastomeric fibre mat reported in ref. 38. Additionally, we further studied its elastic property by stretching it to a predefined strain $(\varepsilon=50 \%)$ for 1,50 and 100 loading and
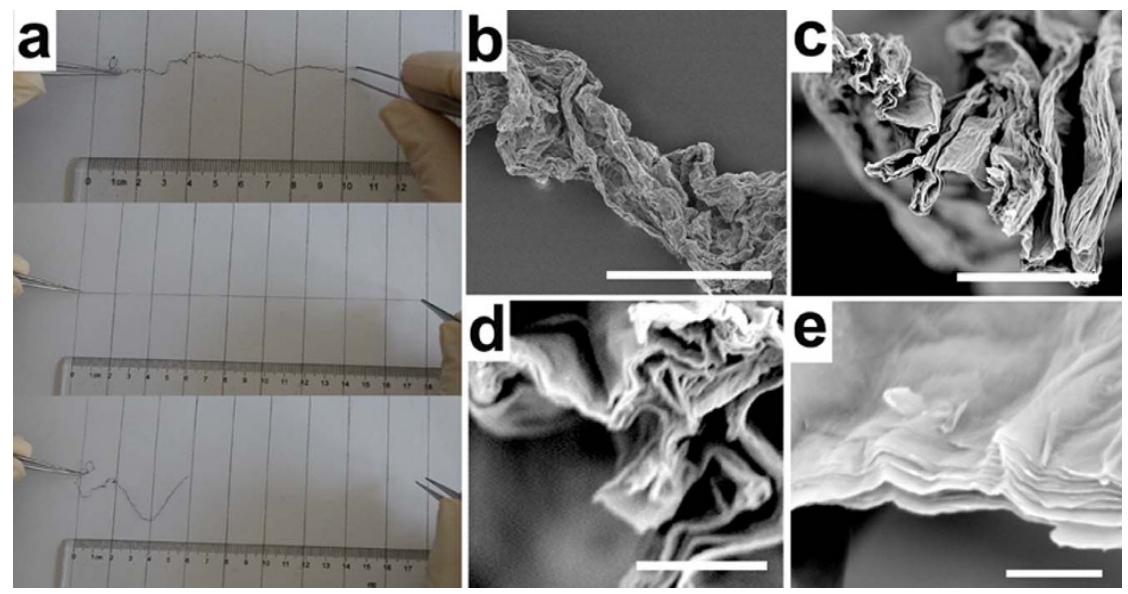

Figure $3 \mid$ (a) Stretching of EGRs from their initial length to a maximum and releasing to free state. FE-SEM images of (b) surface and (c) fracture dregion of an EGR, respectively. (d) and (e) High-magnification images of (c). Scare bars: $200 \mu \mathrm{m}, 50 \mu \mathrm{m}, 5 \mu \mathrm{m}$ and $2 \mu \mathrm{m}$, respectively. 

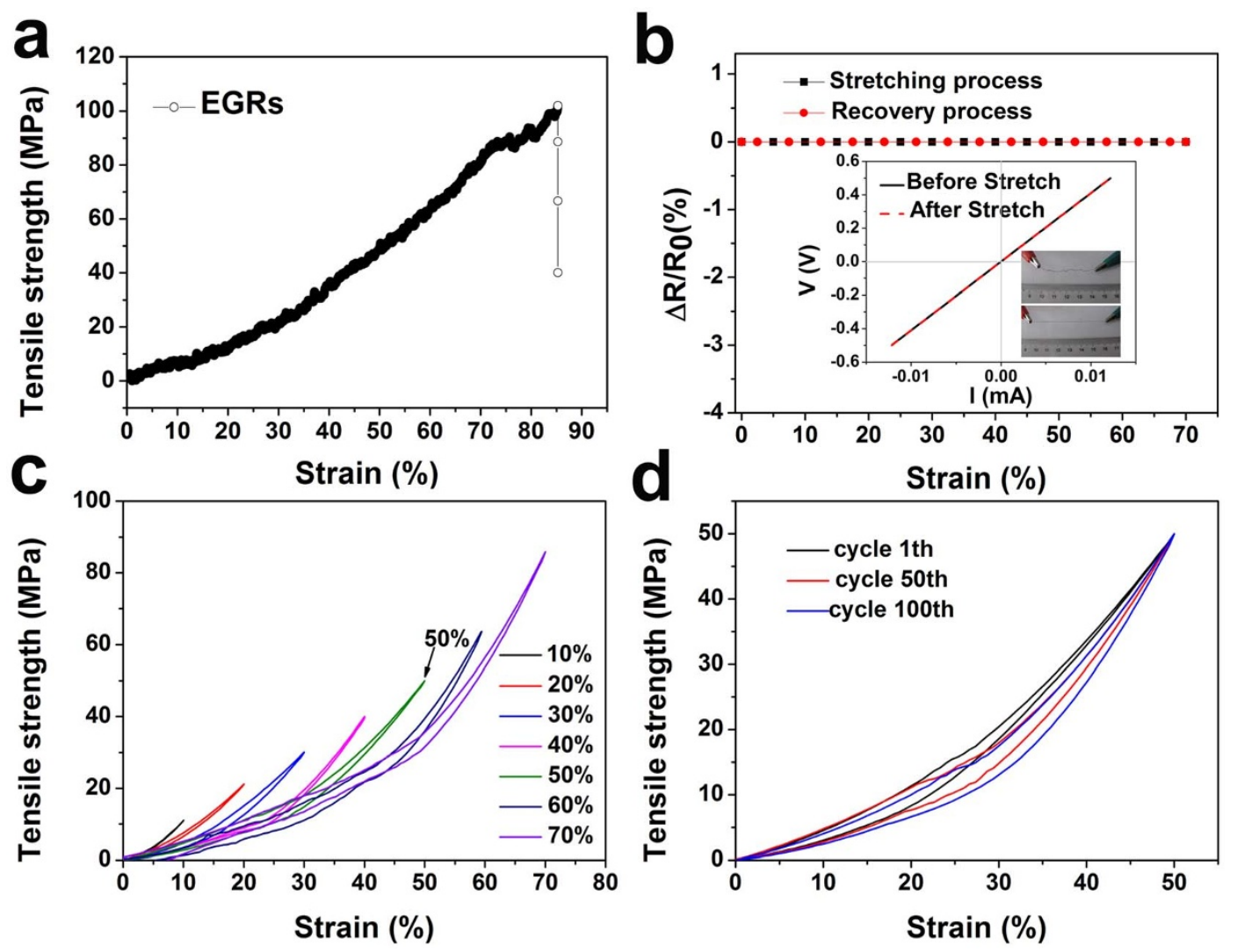

Figure $4 \mid$ (a) The stress-strain curve of an EGR. (b) Relative resistance changes $\left(\Delta R / R_{0}\right)$ of a 4 -cm-long EGR during stretching process $($ black $)$ and recovery process (red), inset shows typical I-V curves of the EGR before (black) and after (red) stretching, they are overlapped. (c) Cyclic stress-strain curves of the EGRs within different strain of $10 \%-70 \%$. (d) Elastic stretching of the EGR within a strain of 50\% (selected cycle 1th, 50th, and 100th are plotted).

unloading cycles. The corresponding cyclic stress-strain curves were showed in Fig. 4d. The result indicates that the elastic stretching remained stable and showed a negligible variation after 100 cycles. The good elastic property of the EGR at room temperature could be attributed to the mixing of disordered "defects" and ordered layer structure, and might be further improved by optimization of the fabrication process. The EGRs exhibited high electronic conductivity, tensile strength, and ultimate strain. Therefore we suppose that they may provide a platform for electronic skins and wearable electronics, sensors, and energy devices.

\section{Discussion}

The electrical and mechanical properties of the GFs, GRs, and EGRs could potentially be further improved by optimizing the initial GO/
$\mathrm{NaDC}$ ratio in hydrogel precursors. The effects of NaDC content (5-9\%) on tensile strength, ultimate failure strain, and electrical conductivity of our graphene-based fibres are shown in Fig. 5 a-c. The most strong and conductive samples were found to be GRs with $8 \%$ and $7 \%$ initial NaDC content, respectively. EGRs exhibited best elasticity (ultimate strain $~ 90 \%$ ) at $9 \%$ initial NaDC content. Based on above results, we believe that there are still rooms for improvement in elastic behaviors of graphene-based fibres. The next grant challenge could be assembly of GO sheets into complex and controllable structures.

In conclusion, we reported a hydrogel-assisted spinning method for fabricating GFs with favorable electrical and mechanical properties. A double-roller crusher was further used to create GRs and EGRs with a long-range ordered structure, to improve the electrical
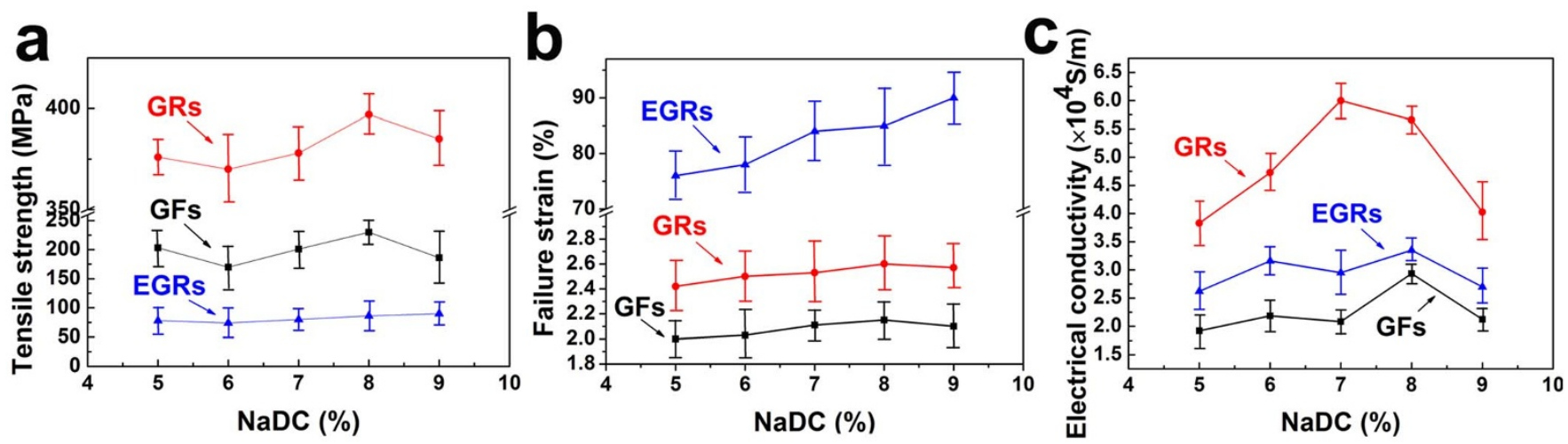

Figure 5 Effects of NaDC content on (a) tensile strength, (b) failure strain, and (c) electrical conductivity of GFs (black), GRs (red), and EGRs (blue). Error bars are the s.d. calculated from repeated measurements. 
and mechanical properties. The rigidity and elasticity of these ribbons could be tuned by controlling the microscopic structure in preparation. EGRs with high elasticity and elastic recovery, as well as enhanced electrical properties were prepared. The electrical resistance of the EGRs does not change during stretching. The GFs, GRs, and EGRs have great potential in flexible/wearable sensors and energy devices.

\section{Methods}

Synthesis of GO and GO hydrogel. GO was synthesized using the Hummers' method. Typically, natural flake graphite powder $(3 \mathrm{~g})$ was added to $0{ }^{\circ} \mathrm{C} 98 \%$ sulfuric acid $(69 \mathrm{~mL})$ in a $500-\mathrm{mL}$ flask. $\mathrm{KMnO}_{4}(9 \mathrm{~g})$ was gradually added while cooling and stirring in an ice bath. The mixture was stirred at $35^{\circ} \mathrm{C}$ for $2 \mathrm{~h}$. Distilled water $(138 \mathrm{~mL})$ was slowly added, and the temperature was maintained at $<100^{\circ} \mathrm{C}$ for $15 \mathrm{~min}$. The mixture was then poured slowly into $30 \% \mathrm{H}_{2} \mathrm{O}_{2}$ solution $(520 \mathrm{~mL})$. The product was filtered with $10 \%$ aqueous $\mathrm{HCl}(750 \mathrm{~mL})$ to remove metal ions, and was thoroughly diluted with distilled water during filtering. The brown-yellow powder of GO was dried for hybrid preparation. GO hydrogel was synthesized by mixing an aqueous $30 \mathrm{mg} / \mathrm{mL}$ GO suspension and $\mathrm{NaDC}$, in the desired ratio in water (5-9\%). The mixture was magnetically stirred for $24 \mathrm{~h}$, during which time the gel formed.

Fabrication of GOFs, GFs, GRs, and EGRs. GO hydrogel was loaded into a plastic syringe and injected into ethanol at a rate of $20 \mathrm{~mL} / \mathrm{h}$, to remove NaDC. After washing with ethanol, GOFs were collected onto the drum, and dried at $60^{\circ} \mathrm{C}$ for $12 \mathrm{~h}$. Dried GOFs were immersed in aqueous $40 \% \mathrm{HI}$ for $2 \mathrm{~h}$ at room-temperature, followed by washing with ethanol and water, and vacuum drying at $80^{\circ} \mathrm{C}$ for $12 \mathrm{~h}$ to obtain GFs. The GO hydrogel was injected into the PTFE pipeline (inner/external diameter of 300/700 $\mu \mathrm{m}$ ). Then the pipeline was pressed in a double-roller crusher and GO hydrogel was squeezed out into ethanol. The layered GOF structure was obtained. It was subsequently either dried and chemically reduced, or chemically reduced and then dried. Finally, the GRs and EGRs were obtained and collected on drums.

Characterizations and measurements. The morphologies of the as-prepared products were determined at $5.0 \mathrm{kV}$ using a JSM-6700F field emission scanning electron microscopy (FESEM). The electrical resistance of the as-prepared products was measured using a two-probe method by a Zahner electrochemical workstation (Zennium CIMPS-1). Conductivity was calculated based on the length L, area A and resistance $\mathrm{R}$ using the formula, conductivity $=\mathrm{L} /\left(\mathrm{R}^{*} \mathrm{~A}\right)$. Area, $\mathrm{A}$ (calculated by the grid method) of each sample was an average based on the measurements at 3-5 different locations along the fiber's long axis direction. Mechanical property tests of as-prepared products were conducted with an Instron material testing system (Instron 5969) at a strain rate of 1 and $40 \mathrm{~mm} / \mathrm{min}$. The densities of as-prepared products were measured as follow: Tetrabromoethane $\left(\mathrm{Br}_{2} \mathrm{CHCHBr}_{2}\right)$ and carbon tetrachloride $\left(\mathrm{CCl}_{4}\right)$ were mixed in a certain proportion to ensure that the as-prepared samples suspended uniformly in the mixture for at least $4 \mathrm{~h}$ at $20^{\circ} \mathrm{C}$. The density of the mixture, and hence the sample density, was measured using a PZ-B-5 liquid specific gravity balance. Unless otherwise stated, all values were determined from samples with $8 \% \mathrm{NaDC}$ content.

1. Lambert, T. N. et al. Synthesis and characterization of titania-graphene nanocomposites. J. Phys. Chem. C 113, 19812-19823 (2009).

2. Allen, M. J., Tung, V. C. \& Kaner, R. B. Honeycomb Carbon: A review of graphene. Chem. Rev. 110, 132-145 (2010).

3. Rao, C. N. R., Sood, A. K., Subrahmanyam, K. S. \& Govindaraj, A. Graphene: the new two-dimensional nanomaterial. Angew. Chem. Int. Ed. 48, 7752-7777 (2009).

4. Geim, A. K. Graphene: status and prospects. Science 324, 1530-1534 (2009).

5. Park, S. \& Ruoff, R. S. Chemical methods for the production of graphenes. Nat. Nanotechnol. 4, 217-224 (2009).

6. Dikin, D. A. et al. Preparation and characterization of graphene oxide paper. Nature 448, 457-460 (2007)

7. Li, D., Muller, M. B., Gilje, S., Kaner, R. B. \& Wallace, G. G. Processable aqueous dispersions of graphene nanosheets. Nat. Nanotechnol. 3, 101-105 (2008).

8. Eda, G., Fanchini, G. \& Chhowalla, M. Large-area ultrathin films of reduced graphene oxide as a transparent and flexible electronic material. Nat. Nanotechnol. 3, 270-274 (2008).

9. Xu, Z. \& Gao, C. Graphene chiral liquid crystals and macroscopic assembled fibres. Nat. Commun. 2, 571-579 (2011).

10. Dong, Z. L. et al. Facile fabrication of light, flexible and multifunctional graphene fibers. Adv. Mater. 24, 1856-1861 (2012).

11. Cong, H. P., Ren, X. C., Wang, P. \& Yu, S. H. Wet-spinning assembly of continuous, neat, and macroscopic graphene fibers. Sci. Rep. 2, 613-619 (2012).

12. Service, R. F. Electronic textiles charge ahead. Science 301, 909-911 (2003).

13. Rogers, J. A. et al. Paper-like electronic displays: large-area rubber-stamped plastic sheets of electronics and microencapsulated electrophoretic inks. Proc. Natl. Acad. Sci. USA 98, 4835-4840 (2001).
14. Ko, S. H. et al. All-inkjet-printed flexible electronics fabrication on a polymer substrate by low-temperature high-resolution selective laser sintering of metal nanoparticles. Nanotechnology 18, 345202-345209 (2007).

15. Sekitani, T. et al. A rubberlike stretchable active matrix using elastic conductors Science 321, 1468-1472 (2008).

16. Hu, C. G. et al. Graphene microtubings: controlled fabrication and site-specific functionalization. Nano Lett. 12, 5879-5884 (2012).

17. Xu, Z., Sun, H. Y., Zhao, X. L. \& Gao, C. Ultrastrong fibers assembled from giant graphene oxide sheets. Adv. Mater. 25, 188-193 (2013).

18. Xu, Z., Sun, H. Y., Zhao, X. L. \& Gao, C. Highly electrically conductive Ag-doped graphene fibers as stretchable conductors. Adv. Mater. 25, 3249-3253 (2013).

19. Xiang, C. S. et al. Graphene nanoribbons as an advanced precursor for making carbon fiber. ACS Nano 7, 1628-1637 (2013).

20. Xiang, C. S. et al. Large flake graphene oxide fibers with unconventional $100 \%$ knot efficiency and highly aligned small flake graphene oxide fibers. Adv. Mater. 25, 4592-4597 (2013)

21. Jung, Y. J. et al. Aligned carbon nanotube-polymer hybrid architectures for diverse flexible electronic applications. Nano Lett. 6, 413-418 (2006).

22. Hahn, M., Barbieri, O., Gallay, R. \& Kotz, R. A dilatometric study of the voltage limitation of carbonaceous electrodes in aprotic EDLC type electrolytes by charge-induced strain. Carbon 44, 2523-2533 (2006).

23. Ghaffari, M. et al. Aligned nano-porous microwave exfoliated graphite oxide ionic actuators with high strain and elastic energy density. Adv. Mater. 25, 6277-6283 (2013).

24. Tan, Z. Q., Ohara, S., Naito, M. \& Abe, H. Supramolecular hydrogel of bile salts triggered by single walled carbon nanotubes. Adv. Mater. 23, 4053-4057 (2011)

25. Hou, C. Y., Zhang, Q. H., Li, Y. G. \& Wang, H. Z. Graphene-polymer hydrogels with stimulus-sensitive volume changes. Carbon 50, 1959-1965 (2012).

26. Hou, C. Y., Zhang, Q. H., Zhu, M. F., Li, Y. G. \& Wang, H. Z. One-step synthesis of magnetically-functionalized reduced graphite sheets and their use in hydrogels. Carbon 49, 47-53 (2011).

27. Shao, Y. L., Zhang, Q. H., Li, Y. G. \& Wang, H. Z. High-performance flexible asymmetric supercapacitors based on $3 \mathrm{D}$ porous graphene $/ \mathrm{MnO}_{2}$ nanorod and graphene/Ag hybrid thin-film electrodes. J. Mater. Chem. C 1, 1245-1251 (2013).

28. Xu, Z., Zhang, Y., Li, P. G. \& Gao, C. Strong, conductive, lightweight, neat graphene aerogel fibers with aligned pores. ACS Nano 6, 7103-7113 (2012).

29. Vigolo, B. et al. Macroscopic fibers and ribbons of oriented carbon nanotubes. Science 290, 1331-1334 (2000).

30. Li, Y., Kinloch, I. A. \& Windle, A. H. Direct spinning of carbon nanotube fibers from chemical vapor deposition synthesis. Science 304, 276-278 (2004).

31. Zhang, M., Atkinson, K. R. \& Baughman, R. H. Multifunctional carbon nanotube yarns by downsizing an ancient technology. Science 306, 1358-1361 (2004).

32. Ericson, L. M. et al. Macroscopic, neat, single-walled carbon nanotube fibers. Science 305, 1447-1450 (2004).

33. Davis, V. A. et al. True solutions of single-walled carbon nanotubes for assembly into macroscopic materials. Nat. Nanotechnol. 4, 830-834 (2009).

34. Goodby, J. W. et al. Characterization of a new helical smectic liquid crystal. Nature 337, 449-452 (1989).

35. Renn, S. S. \& Lubensky, T. C. Abrikosov dislocation lattice in a model of the cholesteric-to-smectic- A transition. Phys. Rev. A 38, 2132-2147 (1988).

36. Wang, G., Shi, G. Y., Wang, H. Z., Zhang, Q. H. \& Li, Y. G. In situ functionalization of stable 3D nest-like networks in confined channels for microfluidic enrichment and detection. Adv. Funct. Mater. doi: 10.1002/adfm.201301936 (2013).

37. Sun, J. et al. Macroscopic, flexible, high-performance graphene ribbons. ACS Nano 7, 10225-10232 (2013).

38. Park, M. et al. Highly stretchable electric circuits from a composite material of silver nanoparticles and elastomeric fibres. Nat. Nanotechnol. 11, 803-809 (2012).

\section{Acknowledgments}

We gratefully acknowledge the financial support by Natural Science Foundation of China (No. 51172042), High-Tech Research and Development Program of China (2012AA030309), Specialized Research Fund for the Doctoral Program of Higher Education (20110075130001), Science and Technology Commission of Shanghai Municipality (12nm0503900, 13JC1400200), the Program for Professor of Special Appointment (Eastern Scholar) at Shanghai Institutions of Higher Learning,Program for Changjiang Scholars and Innovative Research Team in University (TR2011079, IRT1221), the Fundamental Research Funds for the Central Universities and the Program of Introducing Talents of Discipline to Universities (No.111-2-04).

\section{Author contributions}

Y.L. and H.W. designed and directed the study. G.H., C.H. and M.Z. performed the experiments. G.H., C.H., Y.S., M.Z. and H.W. wrote the main manuscript text. M.Z., Q.Z and Y.L. prepared figures. All authors reviewed the manuscript.

\section{Additional information}

Supplementary information accompanies this paper at http://www.nature.com/ scientificreports

Competing financial interests: The authors declare no competing financial interests. 
How to cite this article: Huang, G.J. et al. Highly Strong and Elastic Graphene Fibres Prepared from Universal Graphene Oxide Precursors. Sci. Rep. 4, 4248; DOI:10.1038/ srep04248 (2014). (c) (1) \&) $\odot$ This work is licensed under a Creative Commons AttributionBy No NonCommercial-NoDerivs 3.0 Unported license. To view a copy of this license, visit http://creativecommons.org/licenses/by-nc-nd/3.0 